\title{
El cuerpo habla, se mueve y adquiere identidad $^{1}$
}

\author{
Nina Calero ${ }^{2}$ \\ Corporación Universitaria Minuto de Dios \\ Adelaida Carreño ${ }^{3}$ \\ Corporación Universitaria Minuto de Dios
}

1 Ponencia presentada para el Diplomado Cuerpo y Expresión en la Formación de la Infancia. Corporación Universitaria Minuto de Dios.

2 IX Semestre de Licenciatura en Educación Artística. Diplomado Cuerpo y Expresión en la Formación de la Infancia

3 IX Semestre de Licenciatura en Educación Artística. Diplomado Cuerpo y Expresión en la Formación de la Infancia 


\title{
El cuerpo habla, se mueve y adquiere identidad
}

\section{Resumen}

El cuerpo es la principal herramienta de comunicación en el niño, el gesto y la postura corporal de quienes le rodean, le permiten comprender su entorno. Desde el nacimiento el niño empieza un proceso de auto- reconocimiento, que debe fortalecerse en los diferentes ámbitos sociales a partir de experiencias corporales, como la emisión de sonidos con su cuerpo, la expresión corporal y el juego como parte fundamental de su condición de niño.

Para el niño moverse en el medio que lo rodea es lo más importante, ya que esta experiencia le permite no solo conocer su cuerpo, sino también comunicarse y formar a partir de las relaciones personales y sociales su propia identidad, es por esta razón, que el reconocimiento de su cuerpo y sus habilidades, hacen parte fundamental de esta experiencia que se llama movimiento. A partir del movimiento de su cuerpo el niño se expresa, vive y adquiere habilidades que le permiten conocer su entorno y desarrollarse tanto física, como cognitiva y socialmente, todo esto utilizando herramientas básicas de su estructura física, como el gesto y la postura corporal en diferentes manifestaciones emocionales.

Palabras clave: Comunicación, cuerpo, expresión corporal, movimiento, identidad, niño, gesto.

\section{The body talks, moves and acquires identity}

\begin{abstract}
The body is the main communication tool in the child, gesture and posture of those around you let you understand their environment; from birth the child begins a process of self-recognition to be strengthened in different social spheres from personal experiences, as the emission of sounds with your body, body language and play a fundamental part of your child's condition.

For the child to move in the surrounding medium is what is the most important, as this experience allows the child not only to know his or her body, but also to communicate and form his or her own identity from the personal and social relationships. It is for this reason that the recognition of his body and his skills make fundamental part of this experience is called movement. From the movement of his body the child expresses, lives and acquires skills that enable him or her to understand their environment and so develop themselves physically, cognitively and socially using basic tools of their physical structure, the gesture and posture in different emotional manifestations.
\end{abstract}

Keywords: Communication; body; body language; motion; identity; child; gesture

\section{O corpo fala, move-se e adquire identidade}

\section{Resumo}

O corpo é a ferramenta principal de comunicação com a criança, o gesto e posição corporal das pessoas circundantes permitem à criança compreender seu entorno; desde o nascimento, a criança começa um processo de auto-reconhecimento que deve se fortalecer nos diferentes âmbitos sociais, a partir de experiências corporais, como a emissão de sons com o corpo, a expressão corporal e o jogo como parte fundamental da sua condição de criança.

Para a criança, se mover no médio que o circunda é o mais importante, já que essa experiência lhe permite não só conhecer seu corpo, mas se comunicar e formar, a partir dessas relações pessoais e sociais, sua própria identidade. É por essa razão que o reconhecimento do seu corpo e suas habilidades fazem parte fundamental dessa experiência que se chama movimento. A partir do movimento do seu corpo, a criança se expressa, vive e obtém habilidades que lhe permitem conhecer seu entorno e se desenvolver tanto física, cognitiva quanto socialmente, tudo isso ao utilizar as ferramentas básicas da sua estrutura física como o gesto, a posição corporal nas diferentes manifestações emocionais.

Palavras chave: Comunicação; corpo; expressão corporal; movimento; identidade; criança; gesto. 


\section{Introducción}

Se dice que en el ser humano, el cuerpo es el instrumento de expresión y comunicación por excelencia. El cuerpo además de ser la parte física del ser humano, es el elemento que permite realizar funciones básicas como desplazarse, comer o dormir, él contiene los pensamientos y emociones que ayudan al desarrollo de la persona, y su comunicación se basa específicamente en el movimiento, el gesto y la emisión de sonidos.

El ser humano tiene una visión y representación específica de sí mismo, a esta representación se le llama cuerpo imaginario, sin embargo, no puede desconocer que tiene a su vez un cuerpo real que hace referencia a las formas, capacidades y características verdaderas del cuerpo. Por lo anterior, es de suma importancia dar un valor significativo a esas características y a las habilidades corporales de los niños desde su individualidad, para que así comprendan que el cuerpo no es solo un objeto de uso cotidiano, sino que con él las personas logran relacionarse, actuar y disfrutar de las emociones que les ofrece el mundo que los rodea.

Como el ser humano tiene la necesidad de comunicarse, utiliza principalmente el lenguaje corporal que le permite transmitir sentimientos, actitudes y sensaciones, pero además de ello, este lenguaje le permite identificar cambios de ánimo, ya que el cuerpo adopta posturas específicas dependiendo de la emocionalidad. Durante los primeros años de vida este es el tipo de comunicación que permite a los padres comprender las necesidades de sus hijos, el gesto, su postura corporal y sus sonidos, como el llanto y el grito, son la base de esa comunicación inicial.

La práctica expresiva del lenguaje corporal le permitirá al niño, con el pasar de los años, descubrir las posibilidades del movimiento de su cuerpo, de su gesticulación y su habla para generar en él la seguridad al comunicarse y relacionarse con el medio que lo rodea, pues finalmente la expresión corporal tiene una doble finalidad, por un lado sirve como base de aprendizajes específicos y por otro, tiene un valor en sí misma, ya que colabora en el desarrollo del bagaje experimental del niño. 


\section{La comunicación de cuerpo}

El gesto y el movimiento como participes activos del lenguaje corporal para la comunicación, Ilevan un proceso de desarrollo que se demarca cronológicamente desde el vientre materno, según Henry Wallon (1993), divide este desarrollo en estadios parecidos a los de Piaget, dejando claro que la evolución del gesto y el movimiento es muy importante en la relación niño-madre, sujeto-medio, fortaleciendo su motricidad que es básica para su manejo físico, intelectual y emocional.

H. Wallon (1993) muestra como el niño en relación con el entorno, mueve su cuerpo y gesticula a partir de la emoción y la necesidad de transmitir algo, en los primeros meses de vida los movimientos corporales son básicos y se limitan a la cabeza, brazos, emoción facial y el grito y poco a poco va comprendiendo también el gesto del otro y la significación del mismo, el tono de voz que se utiliza, diferenciando entre negaciones, afirmaciones y motivaciones.

Así el niño descubre las posibilidades que tiene su cuerpo, y como su gesto y manifestación corporal le lleva a desplazarse y comunicarse en el medio en que se desenvuelve. Después del proceso de auto reconocimiento, el niño adquiere posturas corporales frente a la emoción que desea transmitir, gesticula y habla con su cuerpo y si finalmente hay un lenguaje verbal dadas las condiciones físicas intrínsecas en el ser humano, también hay un lenguaje corporal que no puede desligar de sí mismo, por el contrario se fortalecerá día a día, permitiéndole estructurar su personalidad.

El cuerpo es un instrumento importante para nuestra vida, ya que lo es todo, se constituye como un modo de expresión por medio del movimiento y los recursos fundamentales de comunicación y expresión como el gesto (cuerpo) y el movimiento, de esta forma, el gesto es necesario para la expresión y la comunicación. La expresión por medio del gesto es natural en el niño desde los primeros meses, por otra parte, los diferentes movimientos que realizamos crean mensajes diversos hacia los demás, así se construye un espacio para escuchar el cuerpo, dejarlo hablar por medio de la expresión del gesto, liberar tensiones, esta relación entre personas y su entorno mediada por la sensibilidad se le llama experiencia estética (Marín, 2003).

\section{Posibilidades del cuerpo}

La experiencia estética es un acontecimiento particular en cada persona y es la sensibilidad la que la unifica y caracteriza, definiendo su estructura bajo dos elementos importantes: su contexto social-cultural y el cuerpo del niño y sus sentidos y el contexto social-cultural. Los dos están inmersos en instituciones o escenarios como la familia, la escuela y el estado y también se expresan de diferentes maneras en su vida cotidiana, todo esto mediado por su sensibilidad. 
Esta experiencia estética se transforma con la sensibilidad aplicada desde la percepción, así podemos enseñar a desarrollar los sentidos, es decir, que se logre que las relaciones que establezcan sean con todo el cuerpo y no solo con cada sentido de una forma tradicional. A través de los sentidos, esta experiencia debe ser cercana a los niños y a sus vidas cotidianas, de manera que despierte otra visión más táctil, en donde se pueda sentir la temperatura de la luz, la textura de la sombra, la consistencia o inconsistencia del objeto, desarrollando en los niños ser más piel. Como dice Michel Serres (2002) "la piel despliega la sensibilidad, se estremece, expresa, respira, escucha, ve, ama, recibe, rechaza, mediante esta práctica estética muestra la relación con el mundo y como la historia de su vida habla a través de la experiencia que vive el niño y así es como reintegra todos los sentidos del cuerpo."

Siendo un movimiento la danza se expresa por medio del cuerpo, generalmente acompañado de ritmos musicales, toma en cuenta cinco especiales elementos: ritmo, forma, espacio, tiempo y energía, de esta manera localizamos que esta representación de expresión es el desplazamiento efectuado en el espacio por una o todas las partes del cuerpo del niño, diseñando una forma, impulsado por una energía propia el pulso, con un ritmo determinado, durante un tiempo de mayor o menor duración. El uso predominante de uno u otro de los elementos del movimiento no es siempre igual, cada movimiento es único e irrepetible. El lenguaje corporal habla por sí mismo, por debajo de la conciencia ayuda al niño a manifestar y exteriorizar sentimientos, sensaciones e ideas, le permite comunicar sentimientos, sensaciones o ideas, y desarrolla un sentido estético del movimiento.

A través de la danza se intenta conseguir el dominio y control del propio cuerpo, tomando conciencia de este no solo a nivel físico, sino también como instrumento de comunicación. Las experiencias estéticas actuales como la danza contemporánea o danza teatro interfieren sobre la percepción, el cuerpo y las formas de entender lo que pasa, acudiendo desde lo formal a la palabra, el canto, la música y el teatro, es decir, el movimiento en su más amplia acepción.

En el artículo de Pina Basuch, El Cuerpo Trascedente (Carlos Paolillo 2010), la danzarina manifiesta que el moverse es un acto íntimo y espontaneo, Pina asegura que hay mucho de infantil en su danza-teatro y que solo cuando se es niño es posible, actos como jugar con el agua, ensuciarse y pintarse, movimientos que son propios del niño. Actividades através de las cuales se desarrolla la motricidad, que es la base para el desarrollo físico, intelectual y emocional, por ello es importante que los padres propicien la expresión corporal en los niños, que los incentiven a moverse, a conocer su cuerpo y a tener conciencia del espacio. Jugar con ellos, sobre todo en lugares amplios y seguros, permitirán al niño moverse con autonomía y destreza, también promover el lenguaje gestual a través de juegos de imitación, como representar animales o personajes de un cuento, permite no solo desarrollar el lenguaje comprensivo y expresivo, sino también la iniciativa, la desinhibición, el 
ingenio y la imaginación, favoreciendo el bienestar emocional del niño en su totalidad.

Si bien el cuerpo adquiere posturas específicas para comunicar emociones como enojo, tristeza, alegría entre otras, también tiene la posibilidad de crear sonidos que le permitan expresar sus emociones, el tono de la voz, los golpes que se realiza con las manos y con los pies al estar en movimiento, transmiten la energía e intencionalidad de lo que se quiere comunicar. El sonido está implícito en el ser humano, desde que el feto se encuentra en proceso de gestación, empieza a reconocer sonidos a partir de la semana número 16 y aunque inicialmente reconoce la voz y el corazón de su madre, después tendrá la posibilidad de percibir el mundo sonoro y musical gracias a la estructura física del cuerpo que posee un oído, por lo cual, su cuerpo empieza a experimentar sensaciones producidas por estos sonidos y a su vez empieza a emitir sonidos con su voz, boca, manos y pies hasta convertirse en un instrumento que habla a través de la música, que inicialmente se dará por imitación a los sonidos que percibe, pero que finalmente creara su propia identidad.

Cuando el cuerpo se convierte en un elemento sonoro que le permite al niño expresarse, conocerse y descubrir en él posibilidades rítmicas y de movimiento, se logra un proceso de comunicación, que le ayuda también a aceptarse tal y como es y sacar de sus propias habilidades y destrezas todo el provecho posible, así le da importancia al desarrollo de su cuerpo a partir del movimiento.

\section{Fortalecimiento de la identidad a partir del cuerpo}

El desarrollo humano es todo un sistema, y la motricidad gruesa es una de las áreas que forma parte de su sistema indivisible, el desarrollo físico y psicológico ocurre durante los primeros años de vida; esto quiere decir, que el ser humano sólo tiene una oportunidad de desarrollarse y ésta debe protegerse hasta donde sea posible. Es así, como la educación física es tan importante en la infancia porque se caracteriza por grandes cambios en el desarrollo motor.

Desde el primer año de vida el niño comienza a relacionarse con su entorno, moverse, explorar, conocer el mundo que le rodea, esto ayuda a establecer contactos sociales y al funcionamiento cotidiano. La calidad de esta movilidad puede impulsar o detener su desarrollo, llevarlo a conocer su cuerpo y la organización del mismo, progresivamente ampliará su espacio, logrando así establecer la relación espacio temporal y a realizar sus primeras acciones motrices, aquí es donde los niños(as) adquieren la motricidad gruesa básica: caminar, correr, saltar, escalar, trepar, cuadrupedia, reptar, lanzar y capturar, rodar y golpear. Estas habilidades no solo aparecen por efectos de maduración biológica, sino también de la actividad práctica del niño en el medio que lo rodea. 
Muchos son los factores que están asociados a las características del desarrollo de cada persona, situaciones particulares de la historia de cada individuo, le dan un matiz específico a éste, por esto cuando se va a evaluar el desarrollo de un niño es necesario tener en cuenta y analizar algunos factores que lo afectan, existen estudios donde se pueden distinguir: factores biológicos y factores ambientales los cuales hacen que la forma de desarrollarse de cada persona sea diferente. Según el Dr. Luís Schlackse de la Pontifica Universidad de Chile, entre los factores biológicos se tienen en cuenta aquellos elementos genéticos que están en la estructura biológica del individuo y también aquellos que pudieron afectarlo durante el embarazo, el parto o después de éste (prenatales, perinatales y postnatales); y entre los ambientales, se consideran de gran importancia, la estimulación a la que el niño esté expuesto en sus primeros años, el vínculo afectivo con las personas cercanas, las características de las normas de crianza, factores culturales y socioeconómicos propias del grupo al que pertenece, y las condiciones de la familia como cercanía afectiva y límites y reglas claras, si estos últimos factores están bien encaminados favorecen el desarrollo del individuo, pero pueden tener una incidencia negativa si hacen falta o hay problemas en ellos.

Un retraso en la motricidad gruesa, puede traer consigo consecuencias negativas para las demás áreas del desarrollo de un niño y si no son intervenidos a tiempo, pueden generar en un futuro dificultades en educación física o en juegos que impliquen actividad física, bajo rendimiento en juegos colectivos, por lo que pueden formarse jóvenes que tendrán poco interés por los deportes, e incluso pueden presentar problemas de ubicación espacial y de manejo del cuerpo. Estudios demuestran que se pueden presentar dificultades en el área social, ya que estas actividades físicas implican contactos sociales, y un escaso contacto social con sus compañeros reduce sus posibilidades de participación social fundamental para su desarrollo integral.

El juego es el vehículo ideal para llegar a la expresión con los niños y deberá favorecer el trabajo individual y grupal, respetando el modo de expresión de cada uno, debe ser una herramienta para enseñarles a los niños trabajo en equipo, respeto por el otro. Cuando jugamos, las reglas son una expresión clara de la necesidad de acuerdos, por lo que, el niño descubre el valor de la cooperación y de la negociación, hay que permitir que se manifiesten actitudes de inseguridad, la agresividad, desconfianza, miedos, etc, esto permite analizarlos y canalizarlos, para poder sensibilizarlos hacia valores como son: el respeto la solidaridad, la empatía y la conciencia social, es así como el juego lo tomamos como una educación en la convivencia con el otro, sin olvidar que los juegos son juegos y los niños juegan porque les divierte. 


\section{Conclusiones}

La expresión corporal es una disciplina que debe abarcar el desarrollo integral de los niños, es así, como nosotros como maestros debemos crear pautas y establecer técnicas de expresión, para que los niños puedan comunicarse y entenderse a través del lenguaje corporal, y de esta forma la comunicación con los niños sea una danza entre los niños y el maestro.

La presencia de la expresión y comunicación corporal, en la primera infancia, es muy importante en esas edades. En estos momentos es cuando mejor se puede adaptar la conducta del niño y enseñarle a comunicarse con un lenguaje no verbal, además mediante las sesiones de expresión y comunicación corporal se favorecerá la correcta utilización del cuerpo, de esta manera a medida que los niños conozcan y afirmen hábitos que favorezcan una adecuada utilización de su cuerpo, podrán tener unas conductas más saludables que beneficien su desarrollo. La expresión corporal tiene un doble enfoque: por un lado, mejorar la competencia motriz (que el movimiento sea más creativo y expresivo), y por otro, enriquecer la capacidad de valoración estética del movimiento.

El cuerpo representa un medio de expresión para el niño, por lo que se debe potenciar esta capacidad de expresión corporal, en cualquier respuesta motriz dirigida al conocimiento y utilización del propio cuerpo. Nos hemos dado cuenta de que el lenguaje no verbal es muy importante, ya que fuera del aula no se suele trabajar, y permite a los niños sensibilizarlos acerca de que nos podemos comunicar con nuestro propio cuerpo, sin necesidad de las palabras.

Nosotros como maestros, debemos tener claro que para enseñar expresión y comunicación corporal primero hay que aprender a sentirla, para poder después hacer las clases lo más vivenciales posibles, este es el fin de la expresión corporal, sentir, vivir. 


\section{Referencias}

Farina, C. (2005). "Arte, cuerpo y subjetividad. Estética de la formación y pedagogía de las afecciones", (Tesis doctoral). Departamento de Teoría e Historia de la Educación, Universidad de Barcelona, Barcelona: Ediarte.

Marín, V. (2003). Didáctica de la educación artística. Madrid: Pearson Prentice Hall.

Nieto C. (2014) Los sentidos del feto/ El desarrollo de los sentidos del futuro bebé. Recuperado de: http://www.serpadres.es/embarazo/tu-bebe/sentidos-bebe-percibe-feto.html

Olano R. (1993). La psicología genético-dialéctica de H. Wallon y sus implicaciones educativas. Recuperado de http://books.google.com.co/

Polillo C. (2010). El cuerpo trascendente. Recuperado de http://www.danza.es/multimedia/ revista/pina-bausch-el-cuerpo-trascendente

Romero, P. (1999). Didáctica y expresión, hacia una didáctica de la expresión. Bogotá: Scarg.

Serres, M. (2002). Los cinco sentidos. Ciencia, poesía y filosofía del cuerpo. México: Taurus. 